\title{
EDITORIAL
}

\section{Supplementary data for Powder Diffraction}

The process for submitting supplemental data to the American Institute of Physics (AIP) has recently changed. In the past, when these data were submitted, they were stored on a file transfer protocol site titled http://www.aip.org/ pubservs/epaps.html. All data previously placed on this site will remain accessible from there.

Supplemental data should now be submitted via Peer X-Press (PXP) along with your article and figure files. AIP will assign one digital object identifier (DOI) to the published article. Supplemental material now gets deposited and linked with the DOI for the article, allowing easier access to all material by one click of the mouse. These data are also deposited with ICDD and are subject to the same copyright laws as the article.
To submit supplemental data, simply choose "Supplemental data for production" when uploading those files. It is recommended to use common file types, such as .DOC and .PDF, to provide simplistic retrieval. Once the article is approved, all files are forwarded by the managing editor to AIP.

We at Powder Diffraction hope you will submit all supplemental data along with your article and figure files, as it enhances the information in your article. If you have any questions regarding supplemental data, please feel free to contact me at boris@icdd.com. As always, if you have technical difficulties with PXP, contact pxphelp@aip.org.

Nicole M. Ernst Boris

Managing Editor

\section{Note from Editor-in-Chief}

Please note that supplemental data of the digital form of each experimental diffraction pattern used in a newdiffraction-data paper are now required to be submitted via PXP along with its article and figure files. Submission of the supplemental data of each digital observed XRD pattern used in a crystal-structure article is also encouraged.

Ting C. Huang Editor-in-Chief 